\title{
Confirmation of Sentinel Lymph Node Identity by Analysis of Fine-Needle Biopsy Samples Using Inductively Coupled Plasma-Mass Spectrometry
}

\author{
Alison Beavis, BSc (Hons), ${ }^{1}$ Michael Dawson, $\mathrm{PhD},{ }^{1}$ Philip Doble, $\mathrm{PhD},{ }^{1}$ \\ Richard A. Scolyer, MD, FRCPA, FRCPath, ${ }^{2,3,4}$ Roger Bourne, PhD, ${ }^{7}$ \\ Ling-Xi L. Li, BM, PhD, ${ }^{2}$ Rajmohan Murali, MBBS, FRCPA, 2,3,4 \\ Jonathan R. Stretch, MBBS, FRACS, DPhil, ${ }^{2,6}$ Cynthia L. Lean, PhD, ${ }^{8}$ \\ Roger F. Uren, MD, FRACP, ${ }^{5,9}$ and John F. Thompson, MD, FRACS, FACS ${ }^{2,6}$
}

\footnotetext{
${ }^{1}$ Department of Chemistry, Materials and Forensic Science, University of Technology, Sydney, New South Wales, Australia ${ }^{2}$ Sydney Melanoma Unit and Melanoma and Skin Cancer Research Institute, Sydney Cancer Centre, Royal Prince Alfred Hospital, Camperdown, New South Wales, Australia

${ }^{3}$ Department of Anatomical Pathology, Royal Prince Alfred Hospital, Camperdown, New South Wales, Australia

${ }^{4}$ Discipline of Pathology, The University of Sydney, Sydney, New South Wales, Australia

${ }^{5}$ Discipline of Medicine, The University of Sydney, Sydney, New South Wales, Australia

${ }^{6}$ Discipline of Surgery, Faculty of Medicine, The University of Sydney, Sydney, New South Wales, Australia

${ }^{7}$ Department of Medical Radiation Sciences, Faculty of Health Sciences, The University of Sydney, Sydney, New South Wales, Australia

${ }^{8}$ Cancer Institute New South Wales, Sydney, New South Wales, Australia

${ }^{9}$ Nuclear Medicine and Diagnostic Ultrasound, RPAH Medical Centre, Newtown, New South Wales, Australia
}

\footnotetext{
Received May 15, 2007; accepted October 15, 2007; published online: January 3, 2008.

Address correspondence and reprint requests to: John F. Thompson, MD FRACS FACS; E-mail: john.thompson@smu. org.au

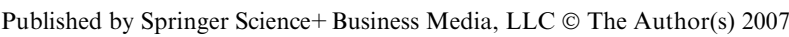


of SLNs, ICP-MS analysis of antimony concentrations in FNB samples could potentially serve as a minimally invasive alternative to surgery and histopathologic evaluation to objectively classify a given node as sentinel or nonsentinel and determine its tumor-harboring status.

Key Words: Clinical—Fine-needle biopsy—Melanoma—Pathology—Sentinel lymph node.

The sentinel lymph node (SLN) biopsy technique, introduced in the early 1990s, has made it possible to establish the tumor-harboring status of the regional node field in melanoma patients with a minimally invasive procedure. ${ }^{1}$ Within 3 years of the publication describing the procedure, the accuracy of SLN biopsy was confirmed in two further studies in which the SLN status was found to accurately reflect the status of the entire lymph node field. ${ }^{2,3}$ The SLN biopsy technique has since become widely accepted as a method of staging the disease of patients with melanoma who have clinically negative lymph nodes. The status of the SLN is used to determine whether further surgical or adjuvant therapy is appropriate, and to assess patient prognosis. The tumor-harboring status of the SLN is the single most important prognostic factor for melanoma patients, surpassing Breslow thickness, ulceration, and mitotic rate. ${ }^{4}$

To provide accurate prognostic information and to guide appropriate management, it is imperative that all "true" SLNs are accurately identified, excised, and assessed for their tumor-harboring status. In a number of cases, a false-negative result may occur, whereby a patient with disease originally assessed as SLN-negative subsequently develops recurrence in a regional node field. It is disturbing to note that falsenegative rates of up to $24.8 \%$ have been reported from some major melanoma treatment centers and cooperative groups. ${ }^{5-16}$ Available evidence suggests that these failures occur not because the SLN biopsy concept is flawed, but because of methodological and technical shortcomings in nuclear medicine, surgery, and histopathology.

In an attempt to identify how many of these falsenegative cases might have been because of surgical failures - that is, the removal of a lymph node that was not the SLN identified by the preoperative lymphoscintigraphy - we have developed a technique for assaying antimony in tissue sections. This work is based on the concept that technetium 99 m-labeled antimony trisulfide colloid $\left({ }^{99} \mathrm{~m}_{\mathrm{Tc}} \mathrm{Sb}_{2} \mathrm{~S}_{3}\right)$ particles used for preoperative lymphoscintigraphy in Australia are retained in the SLN but are absent or present in only tiny amounts in non-SLNs. It has been shown that antimony is preferentially retained by the SLN and can be measured in nodal tissue sections by inductively coupled plasma-mass spectrometry (ICPMS). ${ }^{17}$ It has also been demonstrated that human tissue contains only negligible amounts of antimony. ${ }^{17}$ ICP-MS enables validation of the SLN biopsy procedure ${ }^{26}$ and can identify false-negative results attributable to inaccurate SLN removal. ${ }^{18}$

Although the morbidity associated with SLN biopsy is low, ${ }^{11}$ it is an invasive procedure, with a definite risk of complications and morbidity. ${ }^{19,20}$ In addition, the financial implications of SLN biopsy must be considered. One estimate of the cost of performing a SLN biopsy procedure on an outpatient basis was US\$12,193 ${ }^{21}$ - a high cost for providing melanoma treatment services to the community. The development of a minimally invasive technique for the identification of SLN metastases may minimize or eliminate the morbidity of the procedure and provide a potential cost benefit. It should be possible to identify SLNs by preoperative lymphoscintigraphy, localize the SLNs with ultrasound, and then perform a percutaneous fine-needle biopsy (FNB) under ultrasound control. ${ }^{22}$ Preliminary studies of proton magnetic resonance spectroscopy (MRS) analysis of FNB samples from lymph nodes indicate that this technique has the capacity to distinguish nodes containing metastatic melanoma from uninvolved nodes with high sensitivity, specificity, and accuracy. ${ }^{22,23}$ In addition to accurately determining the tumor-harboring status of the lymph node by this technique, it is also valuable to verify that the sampled lymph node is a "true" SLN, particularly if it has been assessed as being free of metastatic tumor.

The objective of this study was to develop a method for confirming that a FNB specimen was obtained from a true SLN by measuring the level of antimony (present in radiocolloid used during preoperative lymphoscintigraphy) in the FNB sample by ICP-MS.

\section{MATERIALS AND METHODS}

\section{Instrumentation}

All measurements were made with an Agilent 7500ce Inductively Coupled Plasma Mass Spectrom- 
TABLE 1. Operating parameters for Agilent 7500ce Inductively Coupled Plasma-Mass Spectrometry

\begin{tabular}{ll}
\hline Parameter & Condition \\
\hline Radiofrequency power & $1500 \mathrm{~W}$ \\
Sample depth & $8 \mathrm{~mm}$ \\
Plasma gas flow & $15.0 \mathrm{~L} \mathrm{~min}^{-1}$ \\
Carrier gas flow & $1.05 \mathrm{~L} \mathrm{~min}^{-1}$ \\
Peristaltic pump & $.15 \mathrm{rps}$ \\
Sample read delay & $60 \mathrm{~s}$ \\
Rinsing time & $45 \mathrm{~s}$ \\
Dwell time & $100 \mathrm{~ms}$ \\
Replicates & 6 \\
\hline
\end{tabular}

rps, revolution per second.

eter equipped with a MicroMist glass concentric nebulizer, a Quartz-Scott spray chamber (Peltier cooled, $2^{\circ} \mathrm{C}$ ), and an Agilent three-channel peristaltic pump. The operating conditions were optimized daily to ensure maximum sensitivity. Typical operating conditions are summarized in Table 1. The mass spectrometer was operated in spectrum mode with an integration time of $300 \mathrm{~ms}$ on each of the following isotopes: ${ }^{121} \mathrm{Sb},{ }^{123} \mathrm{Sb},{ }^{103} \mathrm{Ru}$ (internal standard/control).

\section{Reagents and Chemicals}

All reagents used were of the highest purity available. Seventy percent double-distilled nitric acid, 37\% hydrochloric acid (Arastar), and 30\% hydrogen peroxide were obtained from Sigma-Aldrich, Australia. Ultra-high-purity water was produced by passing distilled water through a Milli-Q deionizing system (Millipore, Australia).

\section{Standards and Certified Reference Materials}

Certified reference material GBW 07601 Human Hair Powder (Langfang, China) was selected because of its certified antimony levels. Multiple samples of the reference material were also analyzed with each batch of samples and during method development to ensure the accuracy and precision of the analytic technique.

\section{SLN Biopsy Procedure}

Preoperative lymphoscintigraphy was performed to identify the node fields receiving direct lymphatic drainage. This process involved intradermal injec-

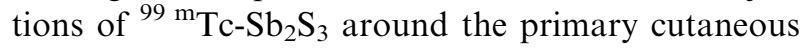
melanoma site, followed by early and delayed imaging with a scintillation camera. ${ }^{24}$ The location of the SLNs was marked on the overlying skin by the nu- clear medicine physician to assist the surgeon in locating SLNs during surgery.

The SLN biopsy procedure was performed within 24 hours of the radiocolloid injection, so that residual radioactivity in lymph nodes could be measured intraoperatively with a handheld gamma probe (Navigator GPS, RMD Instruments, Watertown, MA). Fifteen minutes before the operative procedure, multiple intradermal injections of Patent Blue $\mathrm{V}$ dye (Guerbet, Roissy, France) were made around the primary cutaneous melanoma site. SLN identification was based on visualization of the nodal blue dye staining and results from the preoperative lymphoscintigraphy, with gamma probe confirmation. The experimental protocols of this study were approved by the University of Sydney Ethics Review Board in accordance with the precepts established by the Declaration of Helsinki.

\section{FNB Samples}

The FNB collection process involved puncturing the fresh SLN specimen within 30 minutes of its surgical removal with a 25 -gauge needle attached to a 3-mL plastic syringe. Multiple passes were then made through each quarter of the specimen. A total of 47 FNB samples (from 32 presumptive SLNs and 15 nodes considered to be non-SLNs) were collected. All samples were placed in polypropylene vials containing $300 \mu \mathrm{L}$ of phosphate-buffered saline $(.27 \mathrm{mM}$ of $\mathrm{KCl}, 13.69 \mathrm{mM}$ of $\mathrm{NaCl}, 1.52 \mathrm{mM}$ of $\mathrm{KH}_{2} \mathrm{PO}_{4} ; \mathrm{pH}$ 7.2) made up in perdeuterated water (phosphatebuffered saline- $-\mathrm{D}_{2} \mathrm{O}$ ) and immediately snap-frozen in liquid nitrogen and stored at $-70^{\circ} \mathrm{C}$. $^{22}$

\section{Microwave Digestion Procedure}

Each FNB sample was transferred to a polypropylene tube and prepared by the previously described microwave digestion procedure. ${ }^{17}$ Briefly, the sample was digested in a solution containing $300 \mu \mathrm{L}$ of nitric acid, $300 \mu \mathrm{L}$ of hydrochloric acid, and $500 \mu \mathrm{L}$ of hydrogen peroxide. Each sample was digested five times in a $500 \mathrm{~W}$ microwave oven on the defrost setting for 30 seconds each time. The digest was then quantitatively transferred to a second polypropylene tube, made up to $10 \mathrm{~g}$ with a $1 \%$ nitric acid solution, and assayed by ICP-MS.

\section{Statistical Analysis}

The unpaired $t$-test was used to analyze differences between the levels of antimony in the FNB digests 
TABLE 2. Concentration of antimony ( $p p b$ ) in fine-needle biopsy sample digests collected from sentinel lymph nodes

\begin{tabular}{ll}
\hline Sample No. & Antimony concentration $(\mathrm{ppb})$ \\
\hline 1 & $<$ LOD \\
2 & $<$ LOD \\
3 & $<$ LOD \\
4 & $<$ LOD \\
5 & .0666 \\
6 & .0979 \\
7 & .1738 \\
8 & .2985 \\
9 & .3020 \\
10 & .3085 \\
11 & .3257 \\
12 & .3807 \\
13 & .3812 \\
14 & .3864 \\
15 & .3903 \\
16 & .4045 \\
17 & .4971 \\
18 & .6079 \\
19 & .6407 \\
20 & .7243 \\
21 & .8582 \\
22 & 1.091 \\
23 & 1.438 \\
24 & 1.444 \\
25 & 1.724 \\
26 & 1.760 \\
27 & 1.847 \\
28 & 2.013 \\
29 & 2.150 \\
30 & 2.298 \\
31 & 2.756 \\
32 & 3.248 \\
\hline & \\
\hline & 1.048 \\
\hline
\end{tabular}

LOD, limit of detection (.048 ppb).

from SLNs and non-SLNs. A $P$ value of less than .05 was considered statistically significant.

\section{RESULTS}

The certified concentration of antimony in GBW 07601 is $.095 \pm .012 \mu \mathrm{g} / \mathrm{g}$ (mean $\pm \mathrm{SD}$ ). Replicate analyses of reference materials during method validation and sample analysis gave a mean value of $.088 \pm .007 \mu \mathrm{g} / \mathrm{g}$ and a relative standard deviation of $<9 \%$, confirming the accuracy and precision of the method. The limit of detection (LOD) was evaluated by the $3 \sigma$ criterion (the LOD is given by $m_{b}+3 \sigma_{b}$, where $m_{b}$ is the blank measurement mean and $\sigma_{b}$ is standard deviation of five blank measurements) and found to be .048 parts per billion (ppb).

The matrix-matched calibration standards were in the range of 0 to $20 \mathrm{ppb}$ and gave an $R^{2}$ value of $>.9998$ during method validation and sample analysis. The relative standard deviation of the slopes of the calibration curves was found to be $<10 \%$.
TABLE 3. Concentration of antimony ( $p p b)$ in fine-needle biopsy sample digests collected from nonsentinel lymph nodes

\begin{tabular}{ll} 
Sample No. & Antimony concentration $(\mathrm{ppb})$ \\
\hline 1 & $<$ LOD \\
2 & $<$ LOD \\
3 & $<$ LOD \\
4 & $<$ LOD \\
5 & $<$ LOD \\
6 & $<$ LOD \\
7 & $<$ LOD \\
8 & $<$ LOD \\
9 & $<$ LOD \\
10 & $<$ LOD \\
11 & $<$ LOD \\
12 & $<$ LOD \\
13 & $<$ LOD \\
14 & $<$ LOD \\
15 & .1159 \\
\hline
\end{tabular}

LOD, limit of detection (.048 ppb).

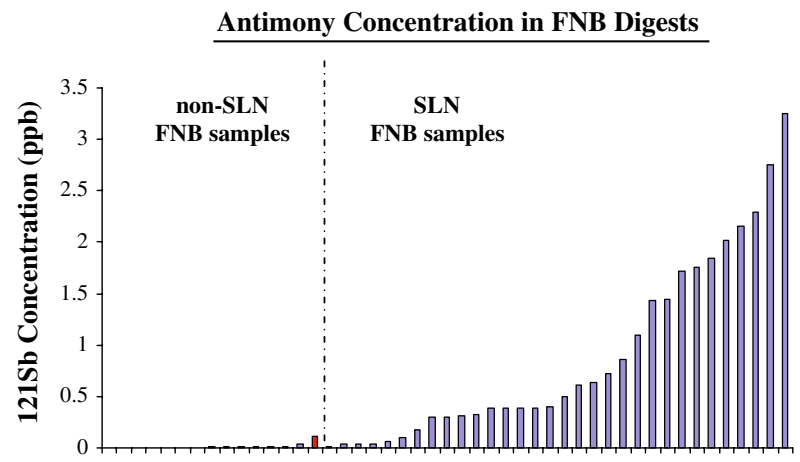

FIG. 1. Antimony concentration in digested fine-needle biopsy samples from sentinel lymph nodes and nonsentinel lymph nodes.

The concentration of antimony in the digested SLNs and non-SLNs was measured, and the results are presented in Tables 2 and 3, respectively. The mean and median concentrations of antimony were .898 and $.451 \mathrm{ppb}$, respectively, in the SLNs (range, $<$ LOD-3.248) and .0145 and .0068 ppb in the nonSLNs (range, <LOD-.1159). These results indicate that the levels of antimony in FNBs from SLNs were significantly greater than from non-SLNs $(P<.00005)$ (Fig. 1).

\section{DISCUSSION}

Current standard clinical management of patients with primary cutaneous melanoma includes wide local excision of the primary tumor and SLN biopsy for patients considered to be at high risk of having regional node field metastases. ${ }^{25}$ Technical failures of 
the procedure could be attributable to errors in lymphoscintigraphy, sentinel lymphadenectomy, or histologic evaluation. In such cases, the potential for disease recurrence in a previously mapped lymph node basin exists. ${ }^{6,8-10,12,14}$

A reliable technique to confirm the identity of a SLN has the potential to reduce false-negative results attributable to surgical errors. In a previous study, we demonstrated that the analysis of antimony in sectioned nodal tissue could be used to distinguish SLNs and non-SLNs removed from the same nodal basin. ${ }^{26}$ This technique can be used within the bounds of currently used histopathologic protocols and has the advantage of being applicable to archival paraffinembedded tissue.

It has also been established that false-negative results may be caused by the removal of a lymph node incorrectly classified as a sentinel lymph node. ${ }^{18} \mathrm{Be}-$ cause neither blue dye nor radioisotope persist after procedures required for microscopic examination, they cannot be used retrospectively to confirm that the "true" SLN had been removed. The only other reported method to confirm SLN identity involves the injection, along with the blue dye, of carbon particles that are retained by the node. ${ }^{27,28}$ However, these large, dense carbon particles may hinder optimal histologic examination by obscuring metastatic melanoma cells. As far as we are aware, this method is not in routine clinical use.

FNB digests from four nodes presumed to be SLNs (13\%) were found to contain extremely low levels of antimony, below the LOD. In a previous study, we also identified SLNs that contained extremely low levels of antimony. ${ }^{26}$ The most likely reason for the low antimony levels would be inaccurate classification of the node that had been removed as a SLN, when it is in fact a non-SLNs. Other possible explanations to account for the low antimony levels include variable distribution of antimony throughout the node, limited migration of the colloid to the lymph nodes, or tumor deposits preventing colloid uptake. Haigh et al. ${ }^{27}$ investigated the distribution of carbon dye in SLNs and found a high concentration of carbon particles around the point of entry of afferent lymphatic channels. It is likely that antimony exhibits an analogous distribution pattern. If so, FNB samples that fail to include this region of the SLN may contain falsely low antimony levels. In view of these factors, it would be useful to set a criterion standard for classifying a given node as sentinel or not on the basis of antimony levels assessed by ICPMS.
However, defining such a criterion on the basis of our small sample set is difficult. In a previous study, we assessed antimony levels in archival tissue sections of paired tumor-positive SLNs and tumor-negative non-SLNs removed from the same regional node field during the same operative procedure from individual patients. ${ }^{26}$ The aims of this study were to determine whether antimony concentrations could be used to confirm whether removed SLNs were "true" SLNs and to differentiate SLNs from non-SLNs. The median concentration of antimony in the SLNs was $.526 \mathrm{ppb}$ and in non-SLNs was $.043 \mathrm{ppb}(P=.004)$. By using a cutoff point of $.18 \mathrm{ppb}$ (the median concentration of antimony in all SLNs and non-SLNs) to differentiate SLNs from non-SLNs, 20 of 24 SLNs and 20 of 24 non-SLNs were correctly identified by the SLN biopsy procedure.

Although SLN biopsy is a highly accurate method for staging regional lymph nodes, it is an invasive surgical procedure that is costly and is associated with an inherent risk of complication and morbidity. ${ }^{19,20}$ Pathologic assessment of SLNs is laborious, time-consuming, and costly, and involves examination of multiple hematoxylin and eosin-stained histopathologic sections as well as sections stained with immunohistochemical techniques. The development of a rapid nonsurgical technique that allows detection of metastatic tumor deposits would be of great benefit. It would eliminate the need for surgical excision of lymph nodes and reduce the complexity of pathologic assessment of SLNs. However, to ensure that potential false-negative results are minimized, it is imperative to ensure the accuracy of SLN identification.

Several studies have identified proton MRS as a candidate for the nonsurgical assessment of lymph nodes. ${ }^{22,23}$ The technique monitors changes in the chemical composition of cells during tumor development and can identify differences that are not morphologically discernible. In these studies, the spectra collected from SLN FNB samples harboring tumor cells contain choline and taurine peaks, which are absent in disease-free samples. ${ }^{23}$ Another sensitive technique for the detection of melanoma metastases in SLNs is the assessment of tyrosinase mRNA by reverse transcriptase-polymerase chain reaction (RTPCR) ${ }^{29,30}$ However, as far as we are aware, RT-PCR assessment of tyrosinase mRNA has not been studied in FNB samples of SLNs. A major potential problem of RT-PCR analysis of FNB samples of SLNs is the issue of false-positive and false-negative results in such specimens and this would need to be assessed before the technique could be used in clinical practice. 
The results of our preliminary work provide evidence that determining antimony levels in FNB specimens by ICP-MS can confirm SLN identity and can differentiate SLNs from non-SLNs. This technique may be a useful adjunct to other techniques assessing tumor-harboring status of SLNs on FNB specimens such as MRS. Potentially, proton MRS analysis of a FNB from a node confirmed to be a true SLN by ICP-MS could provide a reliable method for determining the tumor-harboring status of SLNs. Although the results are promising and raise the possibility that SLN assessment may be performed on FNB specimens (rather than histologic specimens of excised SLNs) in the future, further validation studies are necessary before such techniques are used in widespread clinical practice. Furthermore, the highly specialized and expensive nature of the equipment used for ICP-MS will likely limit the technique to specialist centers.

\section{ACKNOWLEDGMENTS}

R.M. is supported by the Cancer Institute NSW Clinical Research Fellowship Program. The authors thank Chitra De Silva from the Sydney Melanoma Unit for assistance with the collection of samples. Supported in part by the National Health and Medical Research Centre program, grant 402761. Support of the Melanoma Foundation of the University of Sydney is also acknowledged.

\section{OPEN ACCESS}

This article is distributed under the terms of the Creative Commons Attribution Noncommercial License which permits any noncommercial use, distribution, and reproduction in any medium, provided the original author(s) and source are credited.

\section{REFERENCES}

1. Morton DL, Wen D-R, Wong JH, et al. Technical details of intraoperative lymphatic mapping for early stage melanoma. Arch Surg 1992; 127:392-9.

2. Reintgen $\mathrm{D}$, Cruse $\mathrm{CW}$, Wells $\mathrm{K}$, et al. The orderly progression of melanoma nodal metastases. Ann Surg 1994; 220:759-67.

3. Thompson JF, McCarthy WH, Bosch CMJ, et al. Sentinel lymph node status as an indicator of the presence of metastatic melanoma in regional lymph nodes. Melanoma Res 1995; 5:255-60.

4. Morton DL, Thompson JF, Cochran AJ, et al. Sentinel-node biopsy or nodal observation in melanoma. $N$ Engl J Med 2006; 355:1307-17.

5. Cecchi R, De Gaudio C, Buralli L, Innocenti S. Lymphatic mapping and sentinel lymph node biopsy in the management of primary cutaneous melanoma: report of a single-centre experience. Tumori 2006; 92:113-7.

6. Clary BM, Mann B, Brady MS, Lewis JJ, Coit DG. Early recurrence after lymphatic mapping and sentinel node biopsy in patients with primary extremity melanoma: a comparison with elective lymph node dissection. Ann Surg Oncol 2001; 8:328-37.

7. Corrigan MA, Coffey JC, O'Sullivan MJ, Fogarty KM, Redmond HP. Sentinel lymph node biopsy: is it possible to reduce false negative rates by excluding patients with nodular melanoma?. Surg $J$ R Coll Surg E 2006; 4:153-7.

8. Estourgie SH, Nieweg OE, Valdes Olmos RA, Hoefnagel CA, Kroon BBR. Review and evaluation of sentinel node procedures in 250 melanoma patients with a median follow-up of 6 years. Ann Surg Oncol 2003; 10:681-8.

9. Gadd MA, Cosimi AB, Yu J, et al. Outcome of patients with melanoma and histologically negative sentinel lymph nodes. Arch Surg 1999; 134:381-7.

10. Li LX, Scolyer RA, Ka VS, et al. Pathologic review of negative sentinel lymph nodes in melanoma patients with regional recurrence: a clinicopathologic study of 1152 patients undergoing sentinel lymph node biopsy. Am J Surg Pathol 2003; 27:1197-202.

11. Morton DL, Cochran AJ, Thompson JF, et al. Sentinel node biopsy for early-stage melanoma: accuracy and morbidity in MSLT-I, an international multicenter trial. Ann Surg 2005; 242:302-11.

12. Nowecki ZI, Rutkowski P, Nasierowska-Guttmejer A, Ruka W. Sentinel lymph node biopsy in melanoma patients with clinically negative regional lymph nodes - one institution's experience. Melanoma Res 2002; 13:35-43.

13. Rossi CR, De Salvo GL, Trifiro G, et al. The impact of lymphoscintigraphy technique on the outcome of sentinel node biopsy in 1,313 patients with cutaneous melanoma: an Italian Multicentric Study (SOLISM-IMI). J Nucl Med 2006; 47:234 41.

14. Statius Muller MG, Borgstein PJ, Pijpers R, et al. Reliability of the sentinel node procedure in melanoma patients: analysis of failures after long-term follow up. Ann Surg Oncol 2000; 7:4618.

15. van Akkooi AC, de Wilt JH, Verhoef C, et al. High positive sentinel node identification rate by EORTC melanoma group protocol. Prognostic indicators of metastatic patterns after sentinel node biopsy in melanoma. Eur J Cancer 2006; 42:37280.

16. Yee VSK, Thompson JF, McKinnon JG, Scolyer RA, et al. Outcome in 846 cutaneous melanoma patients from a single center after a negative sentinel node biopsy. Ann Surg Oncol 2005; 12:429-39.

17. Dawson M, Doble P, Beavis A, Li LX, et al. Antimony by ICP-MS as a marker for sentinel lymph nodes in melanoma patients. Analyst 2003; 128:217-9.

18. Scolyer RA, Thompson JF, Li LX, et al. Failure to remove true sentinel nodes can cause failure of the sentinel node biopsy technique: evidence from antimony concentrations in falsenegative sentinel nodes from melanoma patients. Ann Surg Oncol 2004; 11(3 Suppl):174S-8S.

19. Wrightson WR, Wong SL, Edwards MJ, et al. Complications associated with sentinel lymph node biopsy for melanoma. Ann Surg Oncol 2003; 10:676-80.

20. Wasserberg N, Tulchinsky H, Schachter J, Feinmesser M, Gutman H. Sentinel-lymph-node biopsy (SLNB) for melanoma is not complication-free. Eur J Surg Oncol 2004; 30:8516.

21. Agnese DM, Abdessalam SF, Burak J, et al. Cost-effectiveness of sentinel lymph node biopsy in thin melanomas. Surgery $2003 ; 134: 542-7$.

22. Stretch JR, Somorjai R, Bourne R, et al. Melanoma metastases in regional lymph nodes are accurately detected by proton 
magnetic resonance spectroscopy of fine-needle aspirate biopsy samples. Ann Surg Oncol 2005; 12:943-9.

23. Lean CL, Bourne R, Thompson JF, et al. Rapid detection of metastatic melanoma in lymph nodes using proton magnetic resonance spectroscopy of fine needle aspiration biopsy samples. Melanoma Res 2003; 13:259-61.

24. Uren RF, Thompson JF, Howman-Giles R, Chung DKV. The role of lymphoscintigraphy in the detection of lymph node drainage in melanoma. Surg Oncol Clin North Am 2006; 15:285-300.

25. Balch CM, Cascinelli N. Sentinel-node biopsy in melanoma. $N$ Engl J Med 2006; 355:1370-1.

26. Scolyer RA, Thompson JF, Li LX, et al. Antimony concentrations in nodal tissue can confirm sentinel node identity. Mod Pathol 2004; 17:1191-7.
27. Haigh PI, Lucci A, Turner RR, et al. Carbon dye histologically confirms the identity of sentinel lymph nodes in cutaneous melanoma. Cancer 2001; 92:535-41.

28. Lucci A, Turner RR, Morton DL. Carbon dye as an adjunct to isosulfan blue dye for sentinel lymph node dissection. Surgery 1999; $126: 48-53$

29. Mangas C, Hilari JM, Paradelo C, Rex J, et al. Prognostic significance of molecular staging study of sentinel lymph nodes by reverse transcriptase-polymerase chain reaction for tyrosinase in melanoma patients. Ann Surg Oncol 2006; 13:910-8.

30. Tatlidil C, Parkhill WS, Giacomantonio CA, Greer WL, Morris SF, Walsh NM. Detection of tyrosinase mRNA in the sentinel lymph nodes of melanoma patients is not a predictor of short-term disease recurrence. Mod Pathol 2007; 20:427-34. 